\title{
WHY PHYTOPHARMACEUTICAL DRUG DISCOVERY?
}

\section{Dear Reader,}

"Phytopharmaceutical drug" includes a purified and standardised fraction with defined minimum fourbio-active orphyto-chemical compounds (qualitatively and quantitatively assessed) of an extract of a medicinal plant or its part, for internal or external use of human beings or animals for diagnosis, treatment, mitigation or prevention of any disease or disorder but does not include administration by parenteral route as specified in Rule 122 (eb) of the Drugs \& Cosmetics (D\&C) Govt. of India". The data requirements have been specified

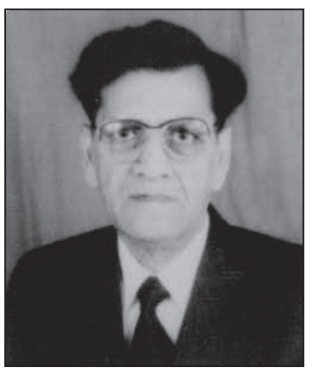
in the Appendix IB of Schedule Y \& GMP manufacturing as per Schedule M (part VI) of D\&C Rules. Clinical trials for phytopharmaceutical drugs is to be conducted as per applicable rules and guidelines for a new drug."

(DCGI promulgated new Phytopharmaceutical drug category notified in the Gazette notification ( $8^{\text {th }}$ Amendment D\&C Rules 2015), Ministry of Health \& Family Welfare, Government of India on $30^{\text {th }}$ November 2015).

Over $50 \%$ of all NCEs approved by the USFDA have its origin in natural phyto-constituents. A successful track record exists of NCEs discovered from medicinal plants over the last 100 years (eg. Quinine, Quinidine, Morphine, Codeine, Reserpine, Colchicine, Camptothecin, Vincristine, Paciltaxol, Artemisinin etc). under well-established regulatory pathways (US-FDA, EMA, DCGI). Presently the NCE pipeline of pharmaceutical MNCs is shrinking drastically. Introduction of the "phytopharmaceutical" category of new drugs opens new vistas for Multi Component Botanical Therapeutics (MCBT) discovery from the rich bio-resource of medicinal \& aromatic plants which have been extensively studied up for pharmacological (in vitro \& in vivo) activities. The identification and characterization of active fractions rather than single chemical constituents leading to bioactive leads in a multicomponent purified fraction of an extract of a medicinal plant has been reported in compilations of research done at various pharmaceutical academic and research institutes in India. An example is the "Reviews on Indian Medicinal Plants" published in 22 volumes covering 5400 Indian medicinal plant species from Alphabet A to $Q$ (as per binomial nomenclature). This is a huge knowledge base available in the country to take forward for a translational approach for new drug discovery and development for a wide variety of therapeutic unmet needs such as Metabolic disorders (pre-diabetes / diabetic complications, osteoarthritis, osteoporosis bronchial asthma), Neurodegenerative disorders (stress, dementia, Alzheimer), Non-alcoholic hepatic fibrotic disorders and Antiviral drugs through phyto-pharmaceutical drug discovery \& development.

One major objective is to investigate the multivalent and multi-target actions of plant constituents present in the active fraction with the aim to understand and rationalize the therapeutic superiority of many phyto-pharmaceuticals over single isolated constituents. Mixtures of interacting constituents produced by plants may provide important combination therapies that simultaneously affect multiple pharmacological targets and provide clinically efficacy beyond the reach of single molecule-based (NCE) drugs. Developing innovative scientific methods for discovery, validation, 
characterization and standardization of these multicomponent botanical therapeutics (MCBT) is essential for their acceptance into main stream medicine.

During the second half of the $20^{\text {th }}$ Century, there had been considerable decline in the number of monographs on plants and plant products in the major Pharmacopoeias of the World such as United States Pharmacopoeia (USP), European Pharmacopoeia (EP) British Pharmacopoeia (BP) and even in Indian Pharmacopoeia (IP). It is heartening to note that during $21^{\text {st }}$ Century there is an upsurge of the re-appearance of a number of monographs on Plants \& Plant Products in such Pharmacopoeias. For example in USP nearly 100, in European Pharmacopoeia 300, in BP over 320 monographs and in Indian Pharmacopoeia over 150 monographs on medicinal/aromatic plants and their products have re-appeared. This exhibits revival and resurgence of interest in drugs from plants.

There is increased awareness for industry-academia partnership in pharmaceutical research. It is noteworthy to mention that certain CSIR research laboratories are endeavoring to contribute to new drug-discovery programs, the Department of Biotechnology is actively engaged in promoting bio-based product development and Indian Council of Medical Research welcomes projects on clinical trials of phyto-pharmaceuticals. All the above mentioned premier scientific research organisations are keen to support new drug discovery from our natural bio-resources and an inter-ministerial expert Committee has been constituted for joint projects on new phyto-pharmaceutical drug discovery. Our Pharmaceutical Industry has exhibited excellent progress in generics and biotechnological products but for innovative new drug discovery, pharmaceutical industry needs to pay much more attention. The promulgation of the new phyto-pharmaceutical drug category and the readiness of all the three premier organizations have created a favourable landscape for pharmaceutical industry to come forward and join this endeavor to new drug discovery through the phyto-pharmaceutical drug route.

\section{About The Guest Editor}

S S Handa, a Pharmaceutical scientist/academician, Ph.D. from London School of Pharmacy, London (1972-75); Postdoctoral (1979-82) at the Department of Pharmacology \& Pharmacognosy, University of Illinois at the Medical Centre, Chicago, USA; served as Professor and Head/Chairman of the University Institute of Pharmaceutical Sciences, Panjab University, Chandigarh (1969-1995) and as Director Indian Institute of Integrative Medicines (IIIM), CSIR (1995-2000). ICS-UNIDO (United Nations) Senior Specialist, "Sustainable Industrial Utilization of Medicinal and Aromatic Plants" (March 2005 - December 2008) at the "United Nations Industrial Development Organization'(UNIDO), International Centre for Science (ICS) \& High Technologies, Area Science Park, Trieste, Italy. Scientific Consultant at Ranbaxy Herbal Drug Research, Ranbaxy Laboratories Ltd (2001-2009). Scientific Adviser at Zandu Pharmaceutical Works, Mumbai (2000 to 2007) and then was on its Board of Directors (2007-2008). Scientific Consultant Emami Group Health Care since 2009 till date. Consultant Glaxo Smith Kline (GSK) Asia Pacific (2010-17).

He has also served on various national and international committees involved in developing standards and policies for the development of phytopharmaceuticals and herbal extracts.

प

If you would like to comment on the editorial please write to us at publications@idmaindia.com 